
\title{
$\begin{array}{ll}\text { Research Square } & \begin{array}{l}\text { Preprints are preliminary reports that have not undergone peer review. } \\ \text { They should not be considered conclusive, used to inform clinical practice, } \\ \text { or referenced by the media as validated information. }\end{array}\end{array}$
}

\section{NCOA3 as a critical oncogene in Thyroid cancer via modulating major signaling pathways}

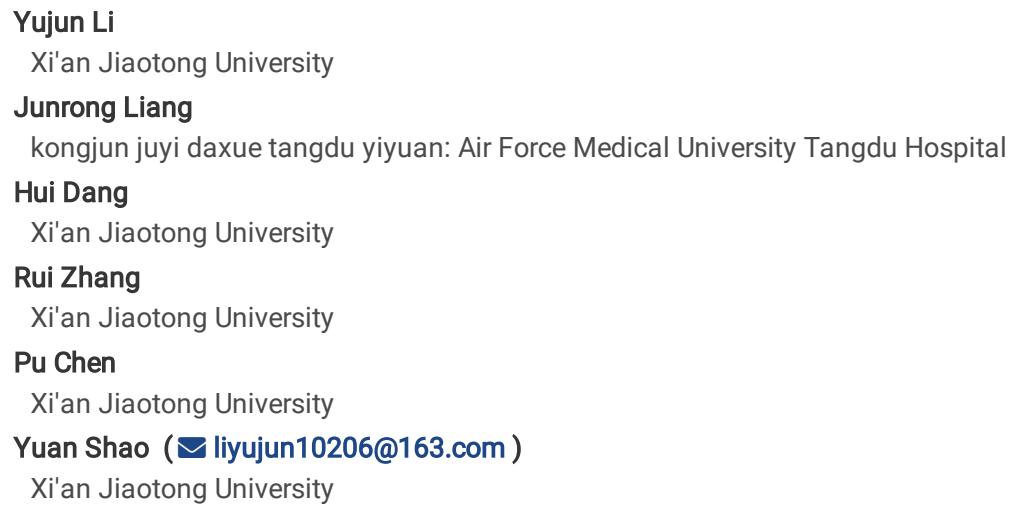

Version of Record: A version of this preprint was published at Endocrine on July 12th, 2021. See the published version at https://doi.org/10.1007/s12020-02102819-6. 


\section{Abstract}

Nuclear Receptor Coactivator (NCOA3) enhances transcriptional activation of nuclear hormone receptors. This study was designed to explore the role of NCOA3 in thyroid cancer. Our data demonstrated that protein expression of NCOA3 was significantly upregulated in thyroid cancer tissues. NCOA3 knockdown inhibited cell proliferation and invasion, and induced cell cycle arrest and apoptosis in thyroid cancer. Conversely, ectopic expression of NCOA3 promoted cell proliferation and invasiveness in thyroid cancer. Mechanically, NCOA3 promotes thyroid cancer cell survival and invasiveness through modulating PI3K/AKT, MAPK and Wnt/ $\beta$-catenin pathways. Collectively, these findings suggest that NCOA3 is critical in the initiation and development of thyroid cancer, and maybe a possible marker for prognostic and therapeutic.

\section{Introduction}

Thyroid cancer is a malignant tumor originated from thyroid follicular epithelial cells, and the incidence of this neoplasia has been increasing in recent years ${ }^{[1}$,

2]. There are several histologic types of thyroid cancer, including papillary, follicular, medullary, and undifferentiated carcinoma. Among them, papillary carcinoma (PTC) is the most common type. Although thyroid cancer can be detected by common diagnostic methods, there are still false negative and undiagnosed cases. Recently, substantial developments have taken place in the translational medicine of thyroid cancer ${ }^{[3,4]}$. The most representative is the elucidation of MAPK/ERK and PI3K/AKT pathways in thyroid tumorigenesis ${ }^{[5]}$. Diagnostic molecular markers have improved risk stratification, and moleculartargeted treatments provide new thoughts for radioiodine-refractory thyroid cancers ${ }^{[6]}$.

NCOA3, Nuclear Receptor Coactivator 3 (also known as SRC-3, AIB1, and ACTR), is a part of the steroid receptor coactivator (SRC) family ${ }^{[7]}$, which enhances transcriptional effects of nuclear receptors and transcription factors such as $\mathrm{E} 2 \mathrm{~F} 1^{[8]}$, nuclear factor-KB (NF-KB) ${ }^{[9]}$ by combined with them. NCOA3 has been discovered as an oncogene in various types of human cancers such as breast cancer ${ }^{[10]}$, prostate cancer ${ }^{[11]}$, and uterine endometrial cancers ${ }^{[12]}$. The exact role of NCOA3 in thyroid tumorigenesis remains largely unknown. Our study found that NCOA3 is ectopic-expressed in thyroid cancer, and plays a tumor-promoting role in thyroid cancer. In addition, our data revealed that NCOA3 down-regulation significantly reduced oncogenic potential of thyroid cancer cells through a network of signaling pathways.

\section{Materials And Methods}

\section{Clinical samples}

21 surgical PTCs and matched non-cancerous tissues were obtained from the First Affiliated Hospital of Xi'an Jiaotong University. Parts of the block were buried in paraffin and the rest were frozen. These patients did not undergo any therapeutic intervention and signed informed consent prior to the operation. All tissues were examined histologically by two senior pathologists from the hospital's pathology department, in accordance with World Health Organization standards.

This study was conducted in accordance with the Helsinki Declaration, and the protocol was approved by the Institutional Review Board and Human Ethics Committee of the First Affiliated Hospital of Xi'an Jiaotong University.

\section{RNA extraction and quantitative RT-PCR (qRT-PCR)}

TRIzol reagent (Takara Inc., Dalian, China) was used to extract total RNA from tissues and cells in accordance with manufacturer instructions, and PrimeScript RT reagent kit (Takara Inc., Dalian, China) was used to produce the cDNA. qRT-PCR was made on the CFX96 DiceTM real-time PCR system (Bio-Rad Laboratories, Inc., CA) using SYBR Premix Ex TaqTM (Takara Inc., Dalian, China). The expression of the mRNA was standardized to cDNA of $18 \mathrm{~S}$ rRNA. Each sample was repeated three times. The sequence of primers was presented in Supplementary Table 1.

\section{Cell lines}

The cell lines of human thyroid cancer K1, FTC133 and 8505C were provided by Dr. Haixia Guan (The First Affiliated Hospital of China Medical University, Shenyang, China). These cells were all grown routinely at $37^{\circ} \mathrm{C}$ in RPMI 1640 or DMEM/Ham with $10 \%$ FBS.

\section{Plasmid constructs and transfection}

To construct NCOA3 expression plasmid, the corresponding open reading frames (ORFs) was amplified by PCR, for 37 cycles of $98^{\circ} \mathrm{C}$ for 10 seconds, $60^{\circ} \mathrm{C}$ for 15 seconds, and $68^{\circ} \mathrm{C}$ for $5 \mathrm{~min}$ and then cloned into the Nhel and Xhol sites of pcDNA3.1(-) vector (Invitrogen, Grand Island, NY), which was designated as pcDNA3.1(-)-NCOA3. The primers used for plasmid construction were shown in Supplementary Table 2. To determine the role of NCOA3 in controlling cell growth and proliferation, we chose $8505 \mathrm{C}$ cells, which showed low-expression of NCOA3. 8505C cells were transfected with the indicated constructs at $70 \%$ confluence using X-tremeGENE HP DNA Transfection Reagent (Invitrogen, Grand Island, NY), with empty vector as control. Geneticin was used for resistance screening of stable transfected cells.

\section{Short interfering RNAs (siRNAs)}

Oligonucleotides of siRNA targeting NCOA3, and control siRNA were produced by Ribobio (Guangzhou, China). The sequences were presented in Supplementary Table 3. Cells were transfected using Lipofectamine 2000 (Invitrogen) with a final siRNA concentration of $50 \mathrm{nM}$.

\section{Cell proliferation, colony formation, cell cycle, apoptosis, migration and invasion assays}


MTT assay and colony formation assay were used to evaluate cell proliferation. Cell cycle distributions and apoptosis were evaluated by flow cytometer. Cell migration and invasion assays were assessed by transwell chambers $(8.0 \mu \mathrm{m}$ pore size; Millipore, MA). The detailed protocols were similarly performed as described previously ${ }^{[13]}$, and each experiment was repeated 3 times.

\section{Western blot analysis}

Cells were lysed in prechilled RIPA buffer. Equivalent amounts of protein lysates was separated and transferred to PVDF membranes (Roche Diagnostics, Mannheim, Germany). Then incubated with the primary antibodies at $4^{\circ} \mathrm{C}$ overnight. The antibody information was presented in Supplementary Table 3. Subsequently, immunobloting signals were visualized using the Western Bright ECL (Advansta, CA) detection system, by incubation with HRP-specific antibodies from ZSGB-BIO.

\section{Animal studies}

4 week old female athymic mice were purchased from SLAC Animal Co., Ltd., and randomly divided into two groups (six /group). Tumor xenografts were established by $6 \times 10^{6}$ FTC133 cells transfected si-NCOA3-709 or si-NC into the right armpit region of nude mice. Tumor volumes were calculated by the formula (length $\times$ width $^{2} \times 0.5$ ) every 2 days. The mice were sacrificed after 15 days, and tumors were removed and weighted. Next, tumors were embedded in paraffin and sectioned at $5 \mu \mathrm{m}$ until use.

All experimental procedures involving animals were approved by the Laboratory Animal Center of Xi'an Jiaotong University and were carried out in accordance with Institution Guidelines.

Immunohistochemistry (IHC)

IHC assay was used to evaluate the expression levels of Ki67 in the xenograft tumors. The protocol was performed as described previously ${ }^{[14]}$.

\section{Statistical analysis}

Gene expression in tumor tissues and control subjects were compared by the two sample $t$ test, and paired samples were compared by the paired t test. All statistical analyses were performed using the SPSS 20.0 software. $P$ values $<0.05$ were considered significant.

\section{Results}

\section{NCOA3 i s overexpressed and associated with poor prognosis in thyroid cancer}

We first tested mRNA levels of NCOA3 in 21 pairs of primary thyroid cancer tissues and paired normal thyroid tissues by quantitative RT-PCR (qRT-PCR) assay. As shown in Fig. 1a, compared with matched normal thyroid tissues, NCOA3 was up-regulated in 11 of 21 (52.3\%) thyroid cancer tissues. Then we randomly selected 4 thyroid cancer tissues to determine protein levels of NCOA3, as shown in Fig. 1b, compared with matched normal thyroid tissues, NCOA3 was upregulated in PTCs. Immunohistochemistry experiment was also performed in 5 randomly selected thyroid cancer tissues, and confirmed this conclusion as shown in Fig. 1c. The protein levels of NCOA3 are up-regulated in PTCs, while it is not the same for mRNA levels, we hypothesized that post-transcriptional mechanisms may be involved in regulating NCOA3 expression. The data were presented as mean \pm SEM. Statistically significant differences were indicated: *, $P<0.05 ; * \star *, P<0.001$.

\section{NCOA3 down-regulation inhibits thyroid cancer cell growth and xenograft tumor growth}

To determine the role of NCOA3 in thyroid tumorigenesis, we used two siRNAs (si-NCOA3-709 and si-NCOA3-2252) to knockdown NCOA3 in K1 and FTC133 cells, which is confirmed by Fig. 2a, and then MTT and colony formation assay were performed. As shown in the left panel of Fig. $2 \mathrm{~b}$, NCOA3 knockdown inhibited cell proliferation and colony formation compared to the control, in si-NCOA3-transfected cells were fewer than those formed in si-NC-transfected cells (Fig. 2c).

NCOA3 knockdown on thyroid cancer cell cycle contributions and apoptosis were also tested. As shown in Fig. 2d, compared to si-NC-transfected cells, cell cycle was arrested at the $\mathrm{G}_{0} / \mathrm{G}_{1}$ phase in si-NCOA3-transfected cells ( $61.2 \pm 1.79$ to $51.7 \pm 0.62$ in $\mathrm{K} 1$ cells, and $57.7 \pm 1.46$ to $51.0 \pm 0.86$ in $\mathrm{FTC} 133$ cells). In addition, as shown in Fig. 2e, we found that si-NCOA3-transfection showed an increase in both early and late apoptosis in comparison with si-NC transfection $(13.4 \% \pm 0.29-7.53 \% \pm 0.33 \%$ in $\mathrm{K} 1$ cells, and $12.8 \% \pm 1.09-7.01 \% \pm 0.53 \%$ in $\mathrm{FTC} 133$ cells).

NCOA3 re-expression in $8505 \mathrm{C}$ cells was confirmed by western blot analysis (Fig. 2f), and ectopic expression of NCOA3 significantly promoted cell proliferation and colony formation compared to the control (Fig. $2 \mathrm{~g}$ and Fig. $2 \mathrm{~h}$ ).

We also assessed the effect of NCOA3 down-regulation on the growth of xenograft tumors in nude mice. As shown in Fig. 3a, compared with xenograft tumors derived from si-NC transfected cells, the ones derived from si-NCOA3 transfected cells grew more slowly. The volume and mean weight xenograft tumors derived from si-NCOA3 transfected cells was signifcantly smaller as compared with si-NC transfected ones $(P=0.003)($ Fig. 3b). Tumor sections were stained for $\mathrm{Ki}-67$ expression to assess the proliferation index. As shown in Fig. $3 \mathrm{c}$, the percentage of Ki-67 positive cells of si-NCOA3 was signifcantly decreased. The data were presented as mean \pm SEM. Statistically significant differences were indicated: ${ }^{*}, P<0.05 ; * \star, P<0.01 ; * \star *, P<0.001$.

\section{NCOA3 down-regulation inhibits thyroid cancer cell migration and invasion}

The effect of NCOA3 knockdown on thyroid cancer cell migration and invasion was also assesed. As shown in Fig. 4a and $4 \mathrm{~b}$, there was significantly smaller amount of migrated and invaded cells in the si-NCOA3-transfected cells than si-NC. To the opposite, As shown in Fig. 4c (left panel), we also found that 
ectopic expression of NCOA3 significantly promoted the ability of cell migration (the upper pannel) and invasion (the lower pannel). Quantitative analysis of colony numbers was shown in the right panel. The data were presented as mean $\pm \mathrm{SEM}$. Statistically significant differences were indicated: $*, P<0.05 ; * \star, P<$ $0.01 ; * \star \star, P<0.001$.

To explore the mechanism of the relationship between NCOA3 and metastatic phenotypes of thyroid cancer cells, we investigated the effect of NCOA3 on the marker of epithelial mesenchymal transition (EMT), which is critical during tumor metastasis including thyroid cancer ${ }^{[15]}$. Immunofluorescence (Fig. $\left.5 \mathrm{a}\right)$ and western blot (Fig. 5b) assay indicated that knocking down NCOA3 in K1 and FTC133 cells substantially increased the expression of E-cadherin (an epithelial cell marker), and also reduced the expression of vimentin (a mesenchymal marker). These results indicated that the EMT process activated by NCOA3 may contribute to metastatic of thyroid cancer. Besides, we observed that NCOA3 knockdown significantly decreased the expression of $\beta$-catenin protein (Fig. 5a and $b$ ). $\beta$-catenin is a key component of the canonical Wnt signaling pathway, and its imbalance in structure can cause metastasis ${ }^{[16]}$. These observation suggests the involvement of NCOA3 in the regulation of Wnt/ $\beta$-catenin pathway, further contributing to thyroid cancer cell growth and metastasis.

\section{NCOA3 modulates major signaling pathways in thyroid cancer}

Thyroid cancer had a greater mutation burden of PI3K/AKT pathway effectors, BRAF and RAS, which were the predominant drivers for proliferation and distant metastases, especilly for PDTC and ATC ${ }^{[4]}$. ErbB receptors was demonstrated to form a positive feedback loop with MAPK pathways and lead to the activation of MAPK/ERK and PI3K/AKT pathways ${ }^{[17]}$. Previous study has demonstrated that ERK1/2 is responsible for Rb phosphorylation ${ }^{[18]}$, which is critical for the regulation of mammalian cell cycle entry ${ }^{[19]}$. We thus want to determine whether oncogenic role of NCOA3 in thyroid cancer is associated with the activation of these pathways by western blot analysis. As shown in Fig. 6a-c, NCOA3 knockdown dramatically decreased the expression of ErbB receptors, phosphorylation of ERK ( $p$-ERK), AKT ( $p-A K T^{S 473}$ and $p-A K T^{T 308}$ ) and Rb (p-Rb) in K1 and FTC133 cell lines as compared with controls. Collectively, these findings suggest that NCOA3 functions as a critical oncogene in thyroid cancer through modulating major signaling pathways.

\section{Discussion}

NCOA3 has histone acetyltransferase activity, also known as AIB-1 (amplified in breast cancer-1), is a nuclear receptor coactivator. We discovered that NCOA3 functions as an oncogene in thyroid tumorigenesis. First, protein levels of NCOA3 were elevated in thyroid cancer tissues compared with control subjects. Second, knocking down NCOA3 significantly inhibited thyroid cancer cell proliferation and metastasis, while ectopic expression of NCOA3 plays a cancerpromoting role. Third, NCOA3 modulates major signaling pathways in thyroid cancer. Our data showed that protein levels of NCOA3 increased in thyroid cancer while mRNA levels were not increased significantly, we assessed that post-transcriptional activation may be involved in regulating NCOA3 expression

${ }^{[20]}$. It is reported that NCOA3 promotes tumorigenesis in a mouse model of thyroid cancer ${ }^{[21]}$, and is essential for ERBB2-driven oncogenesis in mice. while its function in human thyroid cancer cells remains unclear. Thus, we tested the tumorigenic effect of NCOA3 in thyroid cancer. NCOA3 knockdown inhibits proliferation, invasion, and tumorigenic potential of thyroid cancer cells. On the contrary, overexpression of NCOA3 promotes tumor progression. To better understand the oncogenic role of NCOA3 in thyroid tumorigenesis, we also tested the effect on major signaling pathways in thyroid cancer cells. Our results confirmed that NCOA3 silence remarkably inhibited ErbBs and the activity MAPK and PI3K/AKT signaling which is the downstream of ErbBs ${ }^{[22]}$. As previous studies have shown, NCOA3 can directly bind to E2F1 and act as a coactivator of E2F1, is required for E2F1-mediated transcriptional activation ${ }^{[8]}$.Notably, we found that NCOA3 down-regulation significantly decreased phosphorylation of Rb. While Rb phosphorylation has been reported to inhibit transcriptional activation of E2F through the dissociation of the Rb-E2F1 complex ${ }^{[23]}$. We hypothesized that NCOA3 regulates E2F1 in the above direct and indirect ways, thus participating in cell proliferation. In our study, NCOA3 knockdown downregulates $\beta$-catenin, and we hypothetically assumed that NCOA3 directly interacted with transcriptional cointegrator $\mathrm{CBP} / \mathrm{p} 300^{[24]}$, which can activate $\beta$-catenin ${ }^{[25]}$. The above results are shown in Fig. 7, NCOA3 contributes to thyroid carcinogenesis through modulating signaling network, which may represent a potential therapeutic target for thyroid cancer. Efforts have been made to target this oncogene ${ }^{[26]}$. In addition to traditional NCOA3 inhibitors-gossypol and bufalin, a new small molecule inhibitor named as SI-2 and a small molecule stimulator named as MCB-613 capable of targeting NCOA3 has already been tested in animals ${ }^{[26]}$. Since NCOA3 play a major role in thyroid cancer, inhibitors of it can provide sustained benefits to patients who previously had no effective therapeutic options.

\section{Declarations}

\section{Acknowlegment}

This work was supported by the Research Funds of the The First Affiliated Hospital of Xi'an Jiaotong University (2018MS-01).

\section{Declarations}

The authors have declared that no competing interest exists.

\section{Ethics approval}

This study was performed in line with the principles of the Declaration of Helsinki. Approval was granted by the Ethics Committee of Xi'an Jiaotong University (Date 2018/2)

\section{References}


1. F, B., J, F., I, S., RL, S., LA, T., A, J.: Global cancer statistics 2018: GLOBOCAN estimates of incidence and mortality worldwide for 36 cancers in 185 countries. CA: a cancer journal for clinicians 68(6), 394-424 (2018). doi:10.3322/caac.21492

2. Davies, L., Welch, H.G.: Current thyroid cancer trends in the United States. JAMA otolaryngology- head \& neck surgery 140(4), 317-322 (2014). doi:10.1001/jamaoto.2014.1

3. Integrated genomic characterization of papillary thyroid carcinoma. Cell 159(3), 676-690 (2014). doi:10.1016/j.cell.2014.09.050

4. Landa, I., Ibrahimpasic, T., Boucai, L., Sinha, R., Knauf, J.A., Shah, R.H., Dogan, S., Ricarte-Filho, J.C., Krishnamoorthy, G.P., Xu, B., Schultz, N., Berger, M.F., Sander, C., Taylor, B.S., Ghossein, R., Ganly, I., Fagin, J.A.: Genomic and transcriptomic hallmarks of poorly differentiated and anaplastic thyroid cancers. The Journal of clinical investigation 126(3), 1052-1066 (2016). doi:10.1172/jci85271

5. Xing, M.: Molecular pathogenesis and mechanisms of thyroid cancer. Nature reviews. Cancer 13(3), 184-199 (2013). doi:10.1038/nrc3431

6. Xing, M., Haugen, B.R., Schlumberger, M.: Progress in molecular-based management of differentiated thyroid cancer. Lancet (London, England) 381(9871), 1058-1069 (2013). doi:10.1016/s0140-6736(13)60109-9

7. Xu, J., Wu, R.C., O'Malley, B.W.: Normal and cancer-related functions of the p160 steroid receptor co-activator (SRC) family. Nature reviews. Cancer 9(9), 615-630 (2009). doi:10.1038/nrc2695

8. Louie, M.C., Zou, J.X., Rabinovich, A., Chen, H.W.: ACTR/AIB1 functions as an E2F1 coactivator to promote breast cancer cell proliferation and antiestrogen resistance. Molecular and cellular biology 24(12), 5157-5171 (2004). doi:10.1128/mcb.24.12.5157-5171.2004

9. Werbajh, S., Nojek, I., Lanz, R., Costas, M.A.: RAC-3 is a NF-kappa B coactivator. FEBS letters 485(2-3), 195-199 (2000).

10. Anzick, S.L., Kononen, J., Walker, R.L., Azorsa, D.O., Tanner, M.M., Guan, X.Y., Sauter, G., Kallioniemi, O.P., Trent, J.M., Meltzer, P.S.: AlB1, a steroid receptor coactivator amplified in breast and ovarian cancer. Science (New York, N.Y.) 277(5328), 965-968 (1997).

11. Zhou, H.J., Yan, J., Luo, W., Ayala, G., Lin, S.H., Erdem, H., Ittmann, M., Tsai, S.Y., Tsai, M.J.: SRC-3 is required for prostate cancer cell proliferation and survival. Cancer research 65(17), 7976-7983 (2005). doi:10.1158/0008-5472.can-04-4076

12. Sakaguchi, H., Fujimoto, J., Sun, W.S., Tamaya, T.: Clinical implications of steroid receptor coactivator (SRC)-3 in uterine endometrial cancers. The Journal of steroid biochemistry and molecular biology 104(3-5), 237-240 (2007). doi:10.1016/j.jsbmb.2007.03.007

13. Shi, J., Liu, W., Sui, F., Lu, R., He, Q., Yang, Q., Lv, H., Shi, B., Hou, P.: Frequent amplification of AlB1, a critical oncogene modulating major signaling pathways, is associated with poor survival in gastric cancer. Oncotarget 6(16), 14344-14359 (2015). doi:10.18632/oncotarget.3852

14. Shi, J., Qu, Y., Li, X., Sui, F., Yao, D., Yang, Q., Shi, B., Ji, M., Hou, P.: Increased expression of EHF via gene amplification contributes to the activation of HER family signaling and associates with poor survival in gastric cancer. Cell death \& disease 7(10), e2442 (2016). doi:10.1038/cddis.2016.346

15. Han, J., Gao, W., Su, D., Liu, Y.: Silencing of A-Kinase Anchor Protein 4 (AKAP4) Inhibits Proliferation and Progression of Thyroid Cancer. Oncology research 25(6), 873-878 (2017). doi:10.3727/096504016x14783701102564

16. Mulholland, D.J., Dedhar, S., Coetzee, G.A., Nelson, C.C.: Interaction of nuclear receptors with the Wnt/beta-catenin/Tcf signaling axis: Wnt you like to know? Endocr Rev 26(7), 898-915 (2005). doi:10.1210/er.2003-0034

17. Arteaga, C.L., Engelman, J.A.: ERBB receptors: from oncogene discovery to basic science to mechanism-based cancer therapeutics. Cancer cell 25(3), 282303 (2014). doi:10.1016/j.ccr.2014.02.025

18. Seger, R., Krebs, E.G.: The MAPK signaling cascade. FASEB journal : official publication of the Federation of American Societies for Experimental Biology 9(9), 726-735 (1995).

19. Guo, J., Sheng, G., Warner, B.W.: Epidermal growth factor-induced rapid retinoblastoma phosphorylation at Ser780 and Ser795 is mediated by ERK1/2 in small intestine epithelial cells. The Journal of biological chemistry 280(43), 35992-35998 (2005). doi:10.1074/jbc.M504583200

20. Hossain, A., Kuo, M.T., Saunders, G.F.: Mir-17-5p regulates breast cancer cell proliferation by inhibiting translation of AlB1 mRNA. Molecular and cellular biology 26(21), 8191-8201 (2006). doi:10.1128/mcb.00242-06

21. Ying, H., Willingham, M.C., Cheng, S.Y.: The steroid receptor coactivator-3 is a tumor promoter in a mouse model of thyroid cancer. Oncogene $27(6)$, $823-$ 830 (2008). doi:10.1038/sj.onc. 1210680

22. Torres-Arzayus, M.I., Font de Mora, J., Yuan, J., Vazquez, F., Bronson, R., Rue, M., Sellers, W.R., Brown, M.: High tumor incidence and activation of the $\mathrm{PI3K} / \mathrm{AKT}$ pathway in transgenic mice define AIB1 as an oncogene. Cancer cell 6(3), 263-274 (2004). doi:10.1016/j.ccr.2004.06.027

23. Dick, F., Rubin, S.: Molecular mechanisms underlying RB protein function. Nature reviews. Molecular cell biology 14(5), 297-306 (2013). doi:10.1038/nrm3567

24. Chen, H., Lin, R.J., Schiltz, R.L., Chakravarti, D., Nash, A., Nagy, L., Privalsky, M.L., Nakatani, Y., Evans, R.M.: Nuclear receptor coactivator ACTR is a novel histone acetyltransferase and forms a multimeric activation complex with P/CAF and CBP/p300. Cell 90(3), 569-580 (1997).

25. Clevers, H., Nusse, R.: Wnt/beta-catenin signaling and disease. Cell 149(6), 1192-1205 (2012). doi:10.1016/j.cell.2012.05.012

26. Rohira, A.D., Lonard, D.M.: Steroid receptor coactivators present a unique opportunity for drug development in hormone-dependent cancers. Biochemical pharmacology 140, 1-7 (2017). doi:10.1016/j.bcp.2017.04.005

\section{Tables}

Table 1. Sequences of primers used for RT-PCR assays

\begin{tabular}{lcll}
\hline Genes & Gene bank (ID) & Forward primer (5'-3') & Reverse primer (5'-3') \\
\hline NCOA3 & NM_001174087.1 & CCGATTTAAAGCTGAGCTGC & CCAGTCAAAGGATGTTCAAGC \\
$18 S$ & R_003286.2 & CGCCGCTAGAGGTGAAATC & CTTTCGCTCTGGTCCGTCTT \\
\hline
\end{tabular}

Page 5/12 
Table 2. The primers used in this study for plasmid construction

\begin{tabular}{lcc}
\hline Constructs & Forward primer $\left(5^{\prime}-3^{\prime}\right)$ & Reverse primer $\left(5^{\prime}-3^{\prime}\right)$ \\
\hline NCOA3- & ACCCAAGCTGGCTAGATGAGTGGATTAGGAGAAAACTTGG & GTGGCGGCCGCTCGATCAGCAGTATTTCTGATCAGGACCC \\
CDS & NheI \& Xi \\
\hline
\end{tabular}

Table 3. The sequences of siRNAs used in this study

\begin{tabular}{lll}
\hline siRNAs & Sense $\left(5^{\prime}-3^{\prime}\right)$ & Antisense $\left(5^{\prime}-3^{\prime}\right)$ \\
\hline si-NCOA3-709 & GGACCUGGUUAACACAAGUTT & ACUUGUGUUACCAGGUCCTT \\
si-NCOA3-2252 & GGAGGAGUAUCCUCUACAUTT & AUGUAGAGGAUACUCCUCCTT \\
si-NC & UUCUCCGAACGUGUCACGUTT & ACGUGACACGUUCGGAGAATT \\
\hline
\end{tabular}

Table 4. The antibodies used in this study

\begin{tabular}{lll}
\hline Antibodies & Catalog\# & Source \\
\hline anti-GAPDH & M20006 & Abmart \\
anti-SRC3(NCOA3) & Ab2831 & Abcam \\
anti-Ki67 & 550609 & BD Pharmingen \\
anti-total & BS1810 & Bioworld Technology \\
anti-phospho-AKT (Ser473) & BS4007 & Bioworld Technology \\
anti- phospho-AKT (Thr308) & BS4009 & Bioworld Technology \\
anti-total ERK & BS6426 & Bioworld Technology \\
anti-phospho-ERK1/2 & \#4370 & Cell Signaling Technology \\
anti-E-cadherin & ab15148 & Abcam \\
anti-Vimentin & ab8978 & Abcam \\
anti-total-Rb & sc-102 & Santa Cruz \\
anti-phospho-Rb & sc-514031 & Santa Cruz. \\
anti-ErbB1 & sc-101 & Santa Cruz \\
anti-ErbB2 & WL02164 & Wanlei bio \\
anti-ErbB3 & sc-415 & Santa Cruz \\
\hline
\end{tabular}

\section{Figures}

\section{Figure 1}

a

व.
$P=0.2026$

b

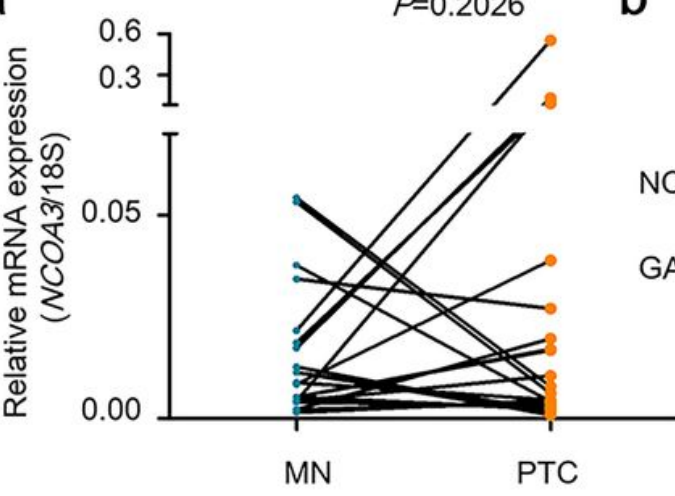

C

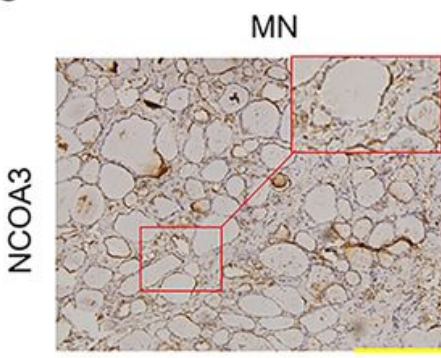

MN PTC MN PTC MN PTC MN PTC

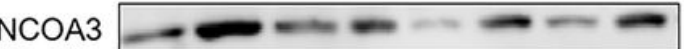

GAPDH

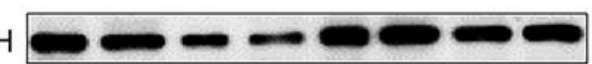

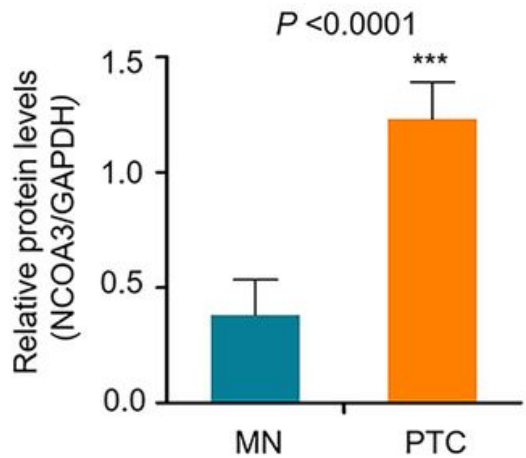

Figure 1 
NCOA3 expression is upregulated in thyroid cancers than in matched non-cancerous thyroid tissues. a, NCOA3 mRNA expression was assessd by qRT-PCR and normalized to 18S rRNA. The data between PTCs and matched none-cancerous tissues (MN) $(n=21)$ were compared using two-tailed paired $t$ test. b, NCOA3 expression in 4 pairs of PTCs and matched non-cancerous tissues (MN) was determined by western blot, and GAPDH was loading control. On the right side of the graph is the density of the corresponding bands of NCOA3 proteins. C, Immunohistochemistry assay of thyroid cancers and para-carcinoma tissues using the antibody of NCOA3. Scale bar, $200 \mu \mathrm{m}$. Statistically significant differences were indicated: ${ }^{*} \mathrm{P}<0.05 ; * \star *, P<0.001$.

Figure 2

a

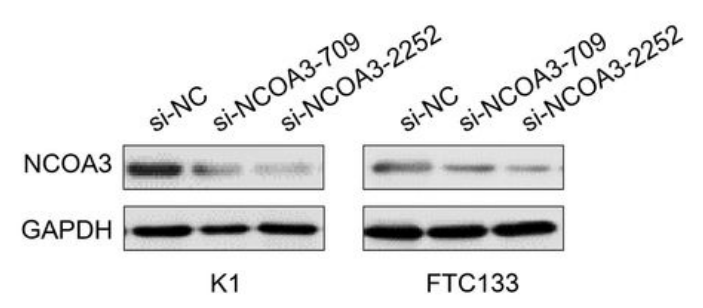

b

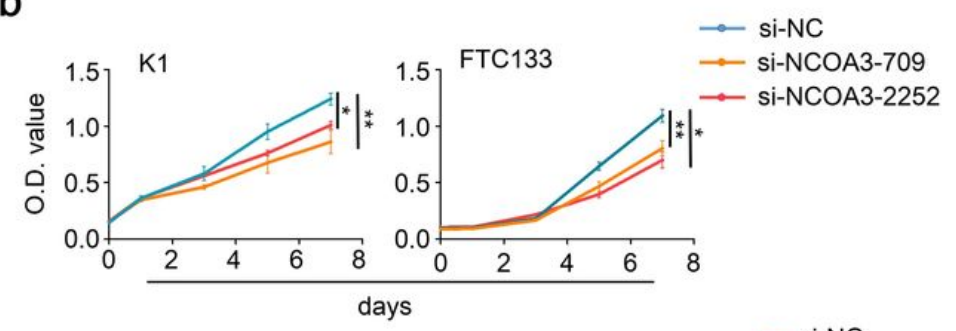

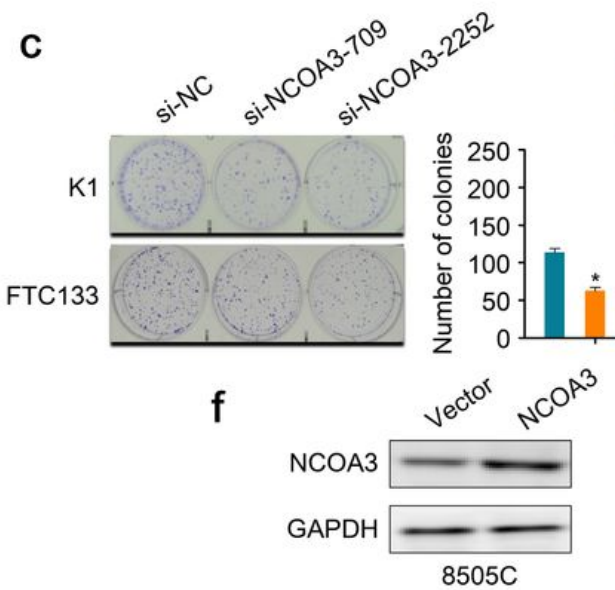

g

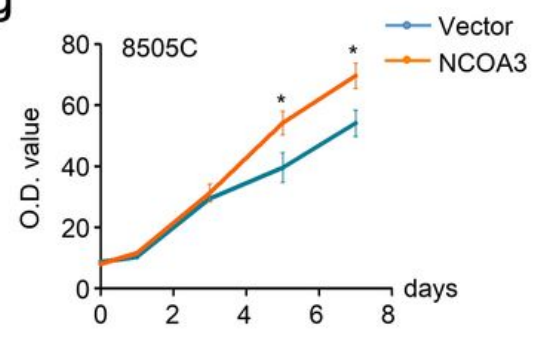

- si-nc d - Si-NCOA3-709 - si-NCOA3-2252

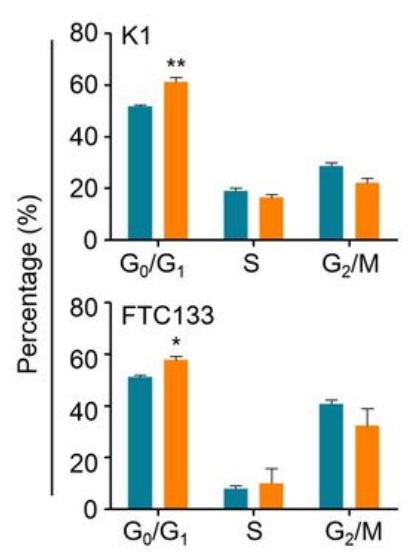

e - si-NC - si-NCOA3-709

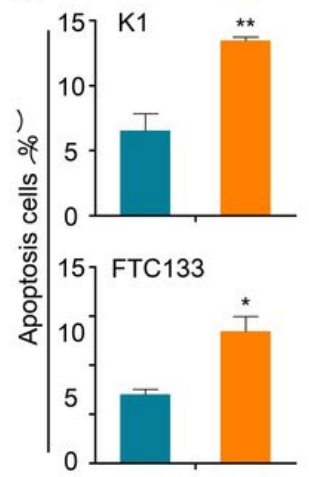

h
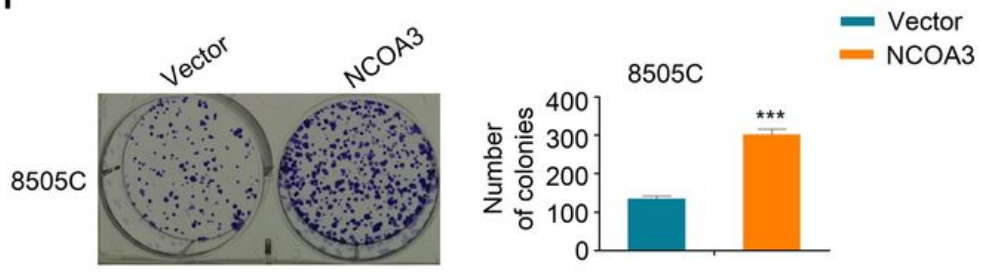

Figure 2

NCOA3 inhibits thyroid cancer cell growth in vitro. a, NCOA3 knock down by two different siRNAs (si- NCOA3-709 and -2252) was evidenced by western blot analysis in $\mathrm{K} 1$ and FTC133 cells. GAPDH was loading control. b, NCOA3 knockdown significantly suppressed thyroid cancer cell proliferation relative to si-NC. c, Knock down of NCOA3 significantly suppressed colony formation in K1 and FTC133 cells. Left panel shows the representative images of colony formation in the transfected cells. Quantitative analysis of colony numbers was shown in right panel. $d$ and e, NCOA3 knock down by si-NCOA3-709 in K1 and FTC133 cells. Cell cycle distribution and apoptosis were then analyzed by flow cytometry. $\mathrm{f}$, NCOA3 was ectopic expressed in 8505C cells, and confirmed by western blot analysis. GAPDH was loading control. g, NCOA3 transfection significantly promoted $8505 \mathrm{C}$ proliferation relative to vector transfection. h, ectopic expression of NCOA3 significantly promoted colony formation in 8505C. Left panel shows the representative images of colony formation. Quantitative analysis of colony numbers was shown in right panel. The data were presented as mean \pm SEM of values from three independent experiments. Statistically significant differences were indicated: *, $\mathrm{P}<0.05$; $* \star, P<0.01$; ***, $\mathrm{P}<0.001$. 
Figure 3

a

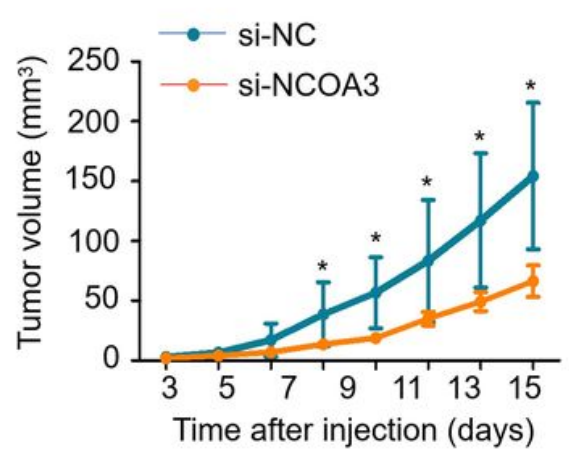

b

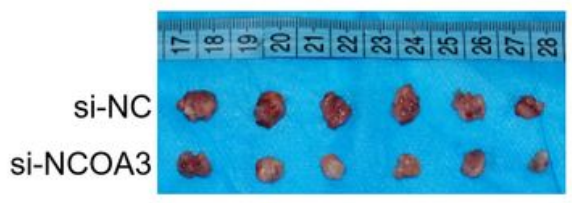

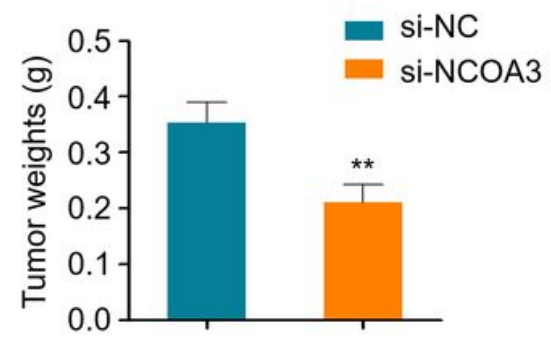

C

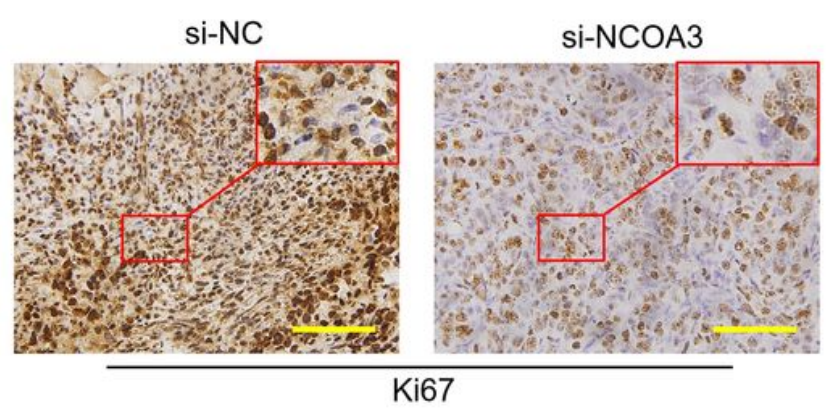

si-NCOA3

i67

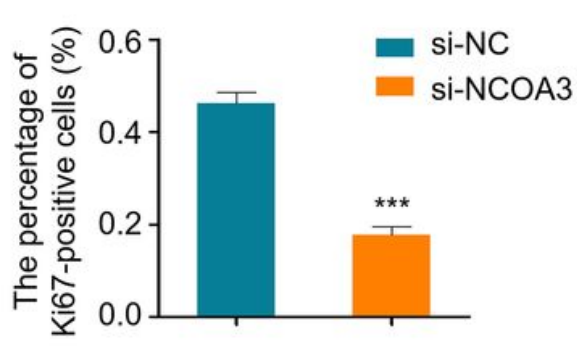

\section{Figure 3}

NCOA3 knockdown inhibits xenograft tumor growth. a, Day 0th represents time point of FTC133 cells transfected si-NCOA3-709 or si-NC injected in nude mice. Tumors were measured, shown as mean \pm SEM ( $n=6$ /group) and curved. $b$, photographs of dissected tumors and Histogram represents mean of tumor weight from nude mice were respectively presented in left panel and right panel. c, shown is representative Ki-67 staining of xenograft tumors from NCOA3knockdown and control mice (left panel). Percentage of Ki-67 positive cells from five microscopic fields in each group represented by histograms in right panel. Scale bar, $200 \mu \mathrm{m}$. The data were presented as mean \pm SEM of values from three independent experiments. Statistically significant differences were indicated: *, $\mathrm{P}<0.05 ; * \star, \mathrm{P}<0.01 ; * \star \star, \mathrm{P}<0.001$. 
Figure 4

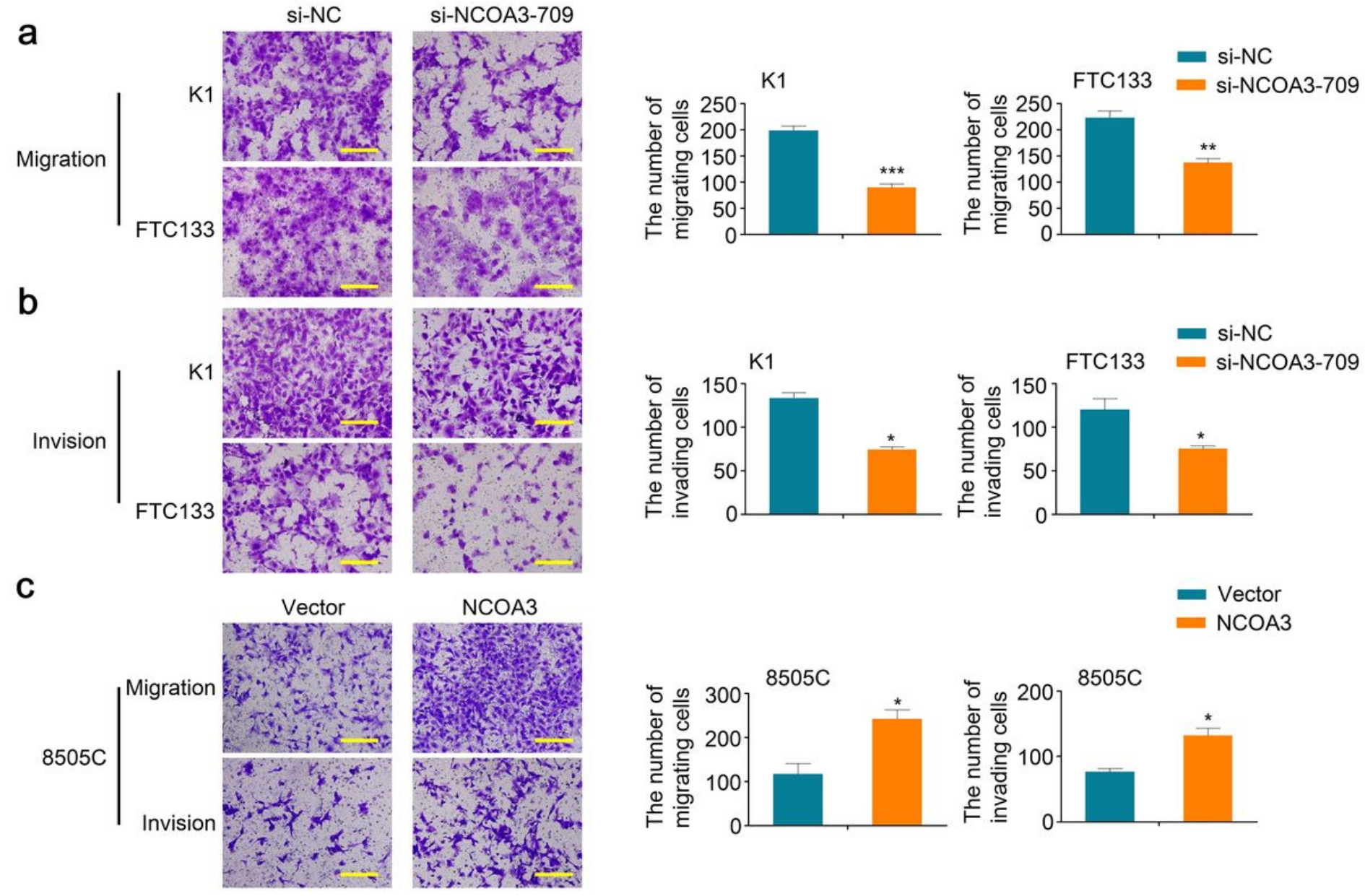

\section{Figure 4}

Effect of NCOA3 on mirgration and invasion. Knock down NCOA3 suppresses migration (upper panels) and invasion (lower panels) of K1(a) and FTC133 cells (b). c, NCOA3 promots migration (upper panels) and invasion (lower panels) of $8505 \mathrm{C}$ cells. The representative images of migrated/invaded cells (left panels). Histograms show means \pm SEM of cell numbers from three independent assays (right panels). Statistically significant differences were indicated: *, $\mathrm{P}<0.05$; $\star *, P<0.01 ; * \star *, P<0.001$. 
Figure 5

a

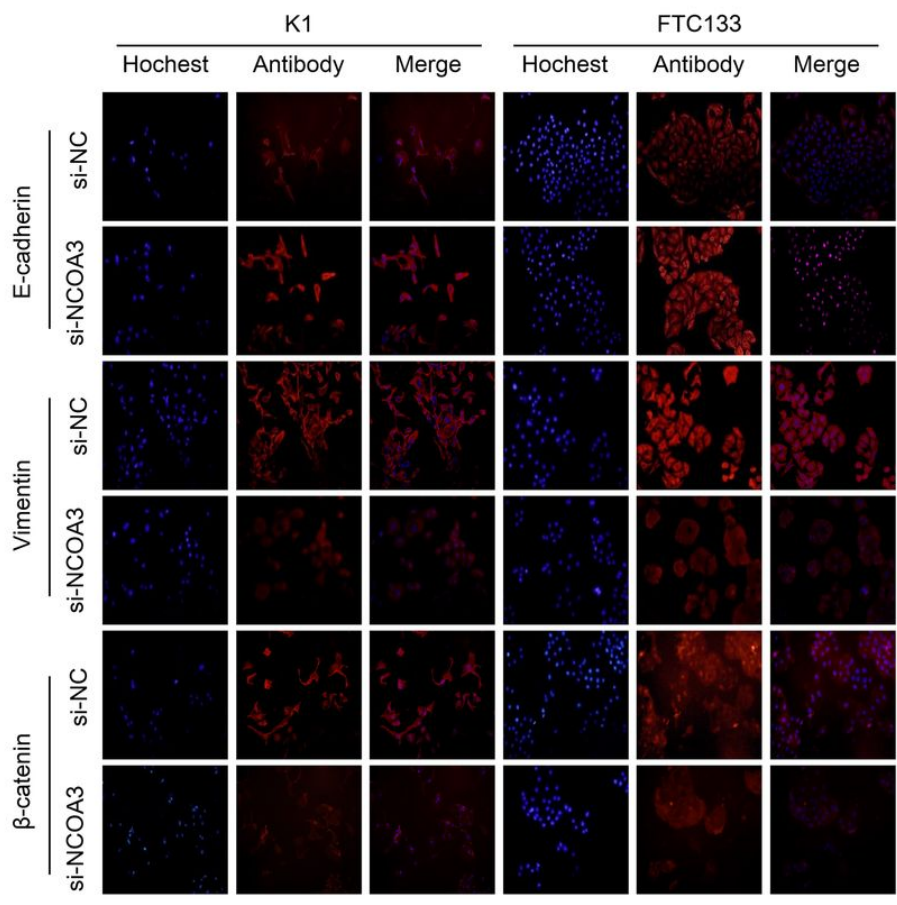

b

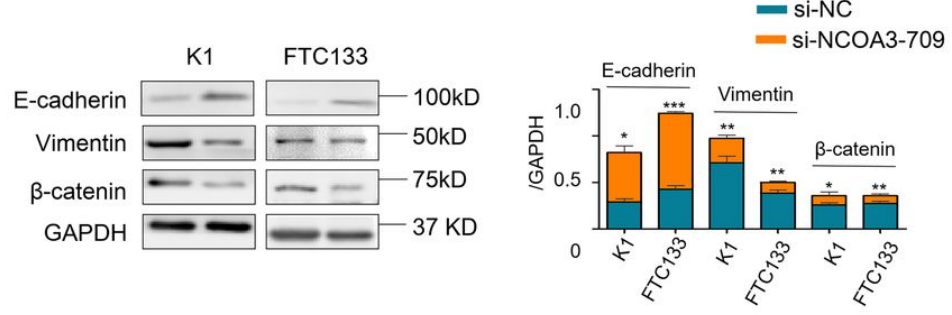

Figure 5

Effect of NCOA3 down-regulation on the expression of E-cadherin, Vimentin and $\beta$-catenin in thyroid cancer cells. a, Immunofluorescence staining was performed to assess the expression of E-cadherin, Vimentin and $\beta$-catenin proteins in K1 and FTC133 cells transfected with si-NCOA3-709 or si-NC. Target protein and Hoechst33342 staining for nuclei were respectively exhibited as red and blue fluorescence. b, western blot analysis was also performed to analyze the three proteins in $\mathrm{K} 1$ and FTC133 cells. GAPDH was loading control. Shown in the right panels is the quantitative illustration of the proteins using densitometry to measure the density of the corresponding in the left panel. Statistically significant differences were indicated: ${ }^{*}, \mathrm{P}<0.05 ; * \star, \mathrm{P}<0.01 ; * \star *, \mathrm{P}$ $<0.001$. 
Figure 6

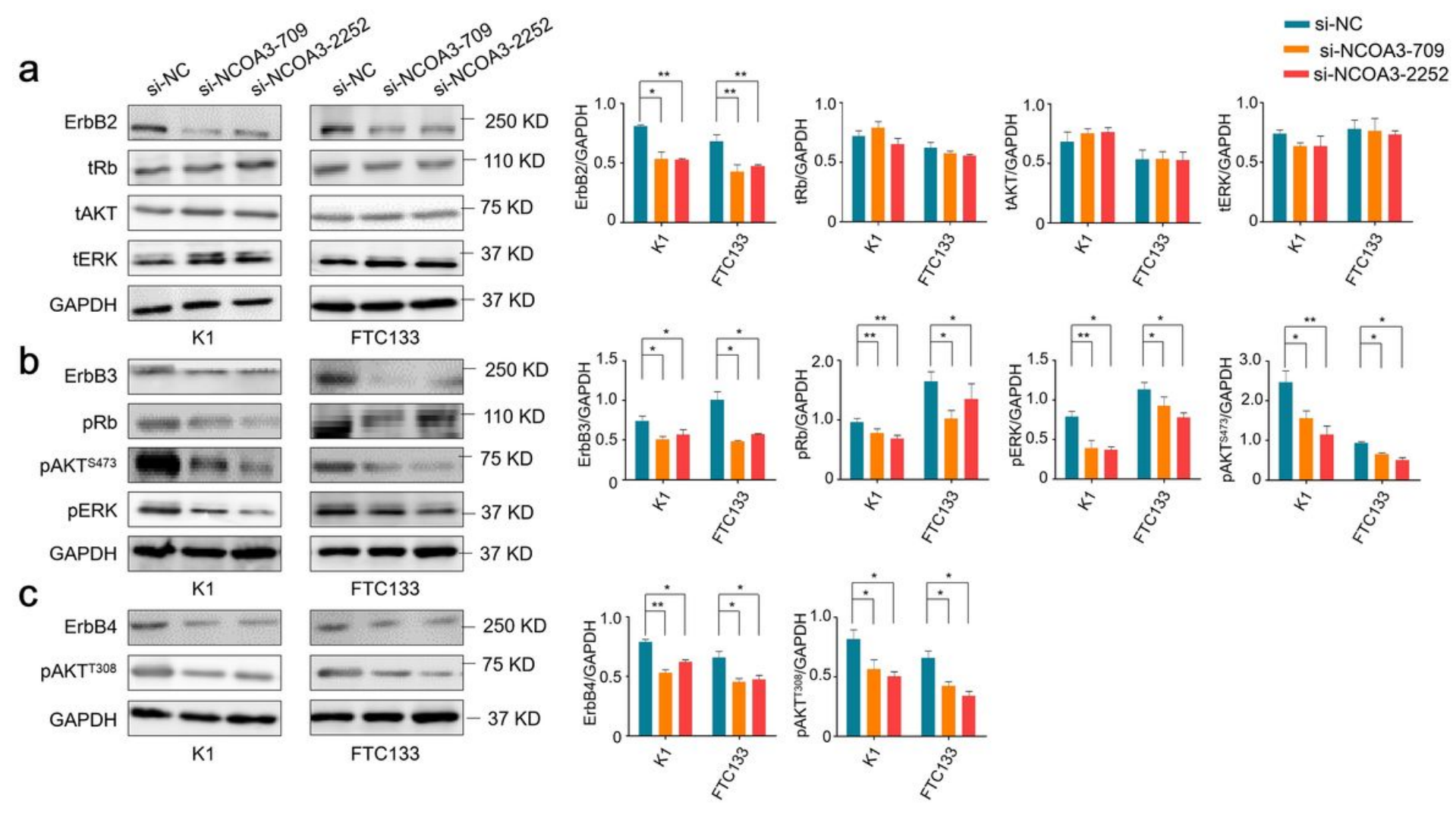

Figure 6

Effect of NCOA3 down-regulation on the expression of ErbB family, Rb, PI3K/AKT and MAPK/Erk pathway in thyroid cancer cells. Cells transfected with siNCOA3-709, si-NCOA3-2252 or si-NC were subjected to western blot assays. a, the antibodies against ErbB2, tRb, total ERK (t-ERK), and total AKT (t-AKT) were used to determine the corresponding protein. $b$, the antibodies against ErbB3, phosphorylated Rb (p-Rb), phospho-ERK (p-ERK), and phospho-AKT S473 ( $p$ AKTS473), c, the antibodies against ErbB4, and phospho-AKT T308 (p-AKT T308) were used to test the effect of NCOA3 down-regulation on ErbB, Rb, MAPK and PI3K/AKT pathways. GAPDH was used as a loading control. Right panels show density of the corresponding bands on Western blot shown in a, b and $\mathrm{c}$. Statistically significant differences were indicated: *, $\mathrm{P}<0.05 ;{ }^{* *}, \mathrm{P}<0.01$. 
Figure 7

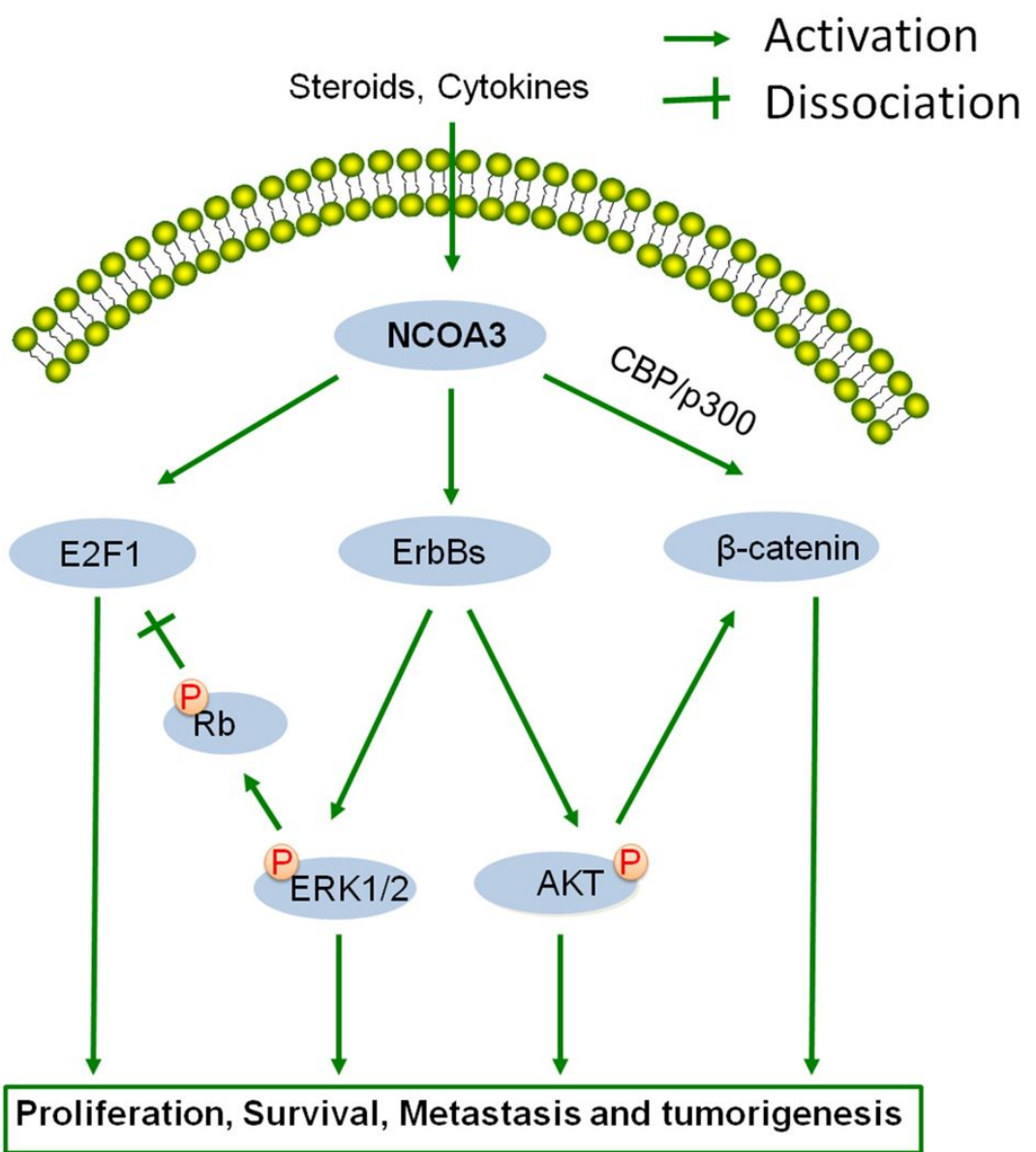

Figure 7

A schematic model of molecular mechanisms underlying oncogenic role of NCOA3 in thyroid cancer. NCOA3 can activates ErbB receptors and its downstream pathways such as MAPK and PI3K/AKT pathways. NCOA3 also promoting phosphorylation of Rb and co-activating E2F1 transcriptional activity. In addition, NCOA3 can activate Wnt/ $\beta$-catenin signaling through different mechanisms. Taken together, NCOA3 overexpression promotes thyroid cancer cell growth and invasiveness through modulating major signaling pathways, ultimately contributing to tumor progression. 